\title{
Enantioselective Guest-Binding of Cationic Ruthenium Complexes by a Chiral Metal-Ligand Assembly
}

Dorothea Fiedler, Dennis H. Leung, Robert G. Bergman, and Kenneth N. Raymond

\section{Supporting Information}

\section{General Considerations}

All reagents were obtained from commercial suppliers and used without further purification unless stated otherwise. Anhydrous solvents were dried over activated alumina. $\quad \mathrm{K}_{6}\left(\mathrm{NMe}_{4}\right)_{5}\left[\mathrm{NMe}_{4} \subset \mathrm{Ga}_{4} \mathrm{~L}_{6}\right] \quad(\mathbf{1 a}), \mathrm{K}_{12}\left[\mathrm{Ga}_{4} \mathrm{~L}_{6}\right] \quad(\mathbf{1 b}), \quad \mathrm{CpRu}(\mathrm{COD}) \mathrm{Cl}$, $\mathrm{CpRu}$ (isoprene) $\mathrm{Cl},[\mathrm{Cp} * \mathrm{RuCl}]_{4}, \mathrm{Cp} * \mathrm{Ru}($ isoprene $) \mathrm{Cl}$ and $\mathrm{Cp} * \mathrm{Ru}(1$-methylbutadiene) $\mathrm{Cl}$

were prepared according to the published procedures. ${ }^{[1-4]}$ All NMR spectra were measured on Bruker AMX 400 or DRX $500 \mathrm{MHz}$ spectrometers. ${ }^{1} \mathrm{H}$ chemical shifts are reported as $\delta$ in ppm relative to residual protonated solvent resonances; ${ }^{13} \mathrm{C}$ chemical shifts are relative to solvent resonances. Coupling constants are reported in Hz. Elemental analyses and were performed by the Microanalytical Laboratory in the College of Chemistry at the University of California at Berkeley. Mass spectra were recorded on VG 70 and triple quadrupole VG Quatttro mass spectrometers at the University of California, Berkeley, Mass Spectrometry facility. Circular Dichroism spectra were measured with a Jasco J-500C spectropolarimeter, equipped with an IF-500 II A/D converter. 


\section{$\underline{\text { Ruthenium complexes }}$}

CpRu(2-ethylbutadiene)Cl. $\quad \mathrm{CpRu}(\mathrm{COD}) \mathrm{Cl} \quad(55.8 \mathrm{mg}, \quad 0.180 \mathrm{mmol})$ was dissolved in $5 \mathrm{~mL}$ abs. EtOH and 2-ethylbutadiene (44.3 $\mathrm{mg}, 0.540 \mathrm{mmol}$ ) was added. The orange solution was heated to reflux for $30 \mathrm{~min}$. The volume of the solution was reduced to $\sim 0.5 \mathrm{~mL}$ and $10 \mathrm{~mL}$ of pentane were added to precipitate the product. The orange powder was isolated by filtration and residual solvent was removed in vacuo. Yield: $84 \%$ (42.9 mg, $0.151 \mathrm{mmol}) .{ }^{1} \mathrm{H}$ NMR (500 MHz, $\left.\mathrm{CD}_{2} \mathrm{Cl}_{2}\right): \delta 5.27\left(\mathrm{dd}, 1 \mathrm{H},{ }^{3} J=\right.$ $\left.10.4 \mathrm{~Hz},{ }^{3} J=7.8 \mathrm{~Hz},=\mathrm{C}-\mathrm{H}\right), 4.90(\mathrm{~s}, 5 \mathrm{H}, \mathrm{Cp}-\mathrm{H}), 4.03\left(\mathrm{dd}, 1 \mathrm{H},{ }^{2} J=1.6 \mathrm{~Hz},{ }^{3} J=7.6 \mathrm{~Hz}\right.$, $\left.=\mathrm{CH}_{2}\right), 3.98\left(\mathrm{~s}, 1 \mathrm{H},=\mathrm{CH}_{2}\right), 2.05\left(\mathrm{~m}, 2 \mathrm{H},-\mathrm{CH}_{2}-\right), 1.31\left(\mathrm{t}, 3 \mathrm{H},{ }^{3} \mathrm{~J}=7.5 \mathrm{~Hz},-\mathrm{CH}_{3}\right), 1.20$ $\left(\mathrm{dd}, 1 \mathrm{H},{ }^{2} \mathrm{~J}=1.6 \mathrm{~Hz},{ }^{3} \mathrm{~J}=10.6 \mathrm{~Hz},=\mathrm{CH}_{2}\right), 1.18\left(\mathrm{~s}, 1 \mathrm{H},=\mathrm{CH}_{2}\right) \mathrm{ppm} .{ }^{13} \mathrm{C}\left\{{ }^{1} \mathrm{H}\right\} \mathrm{NMR}(125$ $\mathrm{MHz}, \mathrm{CD}_{2} \mathrm{Cl}_{2}$ ): 110.1, 86.7 (diene, $\mathrm{sp}^{2}-\mathrm{C}$ ), 84.7 (Cp), 54.4, 53.8 (diene, $\mathrm{sp}^{2}-\mathrm{C}$ ), 32.9, 17.5 (diene, $\mathrm{sp}^{3}$-C) ppm. Anal. Calc. (Found) for $\mathrm{C}_{11} \mathrm{H}_{15} \mathrm{ClRu}$ : C, 46.56 (46.60); H, 5.33 (5.49).

Cp*Ru(2-ethylbutadiene)Cl. $[\mathrm{Cp} * \mathrm{RuCl}]_{4}(85.2 \mathrm{mg}, 78.4 \mu \mathrm{mol})$ was placed in a Schlenk flask. $15 \mathrm{~mL}$ of dry and deoxygenated THF were added to form a red suspension. Via syringe 2-ethylbutadiene $(38.6 \mathrm{mg}, 0.479 \mathrm{mmol})$ was added. The reaction mixture was sirred at room temperature for $1 \mathrm{~h}$ after which it had become a clear orange solution. All volatile materials were removed in vacuo and the resulting dark orange residue was triturated with $3 \times 3 \mathrm{~mL}$ of pentane. The orange, microcrystalline product was collected by filtration and residual solvent was removed under reduced pressure. Yield: $83 \%$ (92.1 mg, $0.260 \mathrm{mmol}) .{ }^{1} \mathrm{H}$ NMR (500 MHz, $\left.\mathrm{CD}_{2} \mathrm{Cl}_{2}\right): \delta 4.43\left(\mathrm{dd}, 1 \mathrm{H},{ }^{3} J=\right.$ $\left.10.2 \mathrm{~Hz},{ }^{3} J=7.6 \mathrm{~Hz},=\mathrm{C}-\mathrm{H}\right), 3.26\left(\mathrm{~s}, 1 \mathrm{H},=\mathrm{CH}_{2}\right), 3.09\left(\mathrm{dd}, 1 \mathrm{H},{ }^{2} J=1.5 \mathrm{~Hz},{ }^{3} J=7.5 \mathrm{~Hz}\right.$, 
$\left.=\mathrm{CH}_{2}\right), 2.12-1.89\left(\mathrm{~m}, 2 \mathrm{H},-\mathrm{CH}_{2}-\right), 1.60\left(\mathrm{~s}, 15 \mathrm{H}, \mathrm{Cp}-\mathrm{CH}_{3}\right), 1.36\left(\mathrm{~s}, 1 \mathrm{H},=\mathrm{CH}_{2}\right) 1.34(\mathrm{t}$, $\left.3 \mathrm{H},{ }^{3} J=7.5 \mathrm{~Hz},-\mathrm{CH}_{3}\right), 1.25\left(\mathrm{dd}, 1 \mathrm{H},{ }^{2} J=1.6 \mathrm{~Hz},{ }^{3} J=10.3 \mathrm{~Hz},=\mathrm{CH}_{2}\right) \mathrm{ppm} .{ }^{13} \mathrm{C}\left\{{ }^{1} \mathrm{H}\right\}$ NMR (125 MHz, $\mathrm{CD}_{2} \mathrm{Cl}_{2}$ ): 113.9 (diene, $\left.\mathrm{sp}^{2}-\mathrm{C}\right), 95.9\left(\underline{\mathrm{Cp}}-\mathrm{CH}_{3}\right), 89.7,53.5,52.9$ (diene, $\left.\mathrm{sp}^{2}-\mathrm{C}\right), 30.1,19.0$ (diene, sp $\left.{ }^{3}-\mathrm{C}\right), 9.7\left(\mathrm{Cp}^{-} \mathrm{CH}_{3}\right)$ ppm. Anal. Calc. (Found) for $\mathrm{C}_{16} \mathrm{H}_{24} \mathrm{ClRu}$ : C, 54.46 (54.56); H, 6.86 (7.02).

Cp*Ru(2-i-propylbutadiene)Cl. A procedure analogous to that employed for $\mathrm{Cp} * \mathrm{Ru}\left(2\right.$-ethylbutadiene)Cl, starting with $80.2 \mathrm{mg}(73.8 \mu \mathrm{mol})$ of $[\mathrm{Cp} * \mathrm{RuCl}]_{4}$ and 42.6 $\mathrm{mg}(0.443 \mathrm{mmol})$ of 2-isopropylbutadiene, led to the formation of the desired product as a dark orange microcrystalline solid in $78 \%$ yield $(84.7 \mathrm{mg}, 0.230 \mathrm{mmol}) .{ }^{1} \mathrm{H}$ NMR (500 $\left.\mathrm{MHz}, \mathrm{CD}_{2} \mathrm{Cl}_{2}\right): \delta 4.36\left(\mathrm{dd}, 1 \mathrm{H},{ }^{3} J=9.8 \mathrm{~Hz},{ }^{3} J=7.6 \mathrm{~Hz},=\mathrm{C}-\mathrm{H}\right), 3.59\left(\mathrm{~s}, 1 \mathrm{H},=\mathrm{CH}_{2}\right), 2.99$ (dd, $1 \mathrm{H},{ }^{2} J=1.7 \mathrm{~Hz},{ }^{3} J=7.5 \mathrm{~Hz},=\mathrm{CH}_{2}$ ), 2.33 (sept, $1 \mathrm{H},{ }^{3} J=6.7 \mathrm{~Hz}, i-\operatorname{Pr} \mathrm{C}-\mathrm{H}$ ), 1.59 (s, 15H, Cp-CH $\left.\mathrm{CH}_{3}\right), 1.37\left(\mathrm{~d}, 3 \mathrm{H},{ }^{3} J=6.9 \mathrm{~Hz}, i-\mathrm{Pr} \mathrm{CH}_{3}\right), 1.35\left(\mathrm{~d}, 1 \mathrm{H},{ }^{2} J=2.0 \mathrm{~Hz},=\mathrm{CH}_{2}\right), 1.22$ $\left(\mathrm{dd}, 1 \mathrm{H},{ }^{2} J=1.7 \mathrm{~Hz},{ }^{3} J=9.9 \mathrm{~Hz},=\mathrm{CH}_{2}\right), 1.06\left(\mathrm{~d}, 3 \mathrm{H},{ }^{3} J=7.2 \mathrm{~Hz}, i-\operatorname{Pr} \mathrm{CH}_{3}\right)$ ppm. ${ }^{13} \mathrm{C}\left\{{ }^{1} \mathrm{H}\right\}$ NMR (125 MHz, $\left.\mathrm{CD}_{2} \mathrm{Cl}_{2}\right): 121.9$ (diene, $\left.\mathrm{sp}^{2}-\mathrm{C}\right), 95.6\left(\underline{\mathrm{Cp}}-\mathrm{CH}_{3}\right), 86.9,51.4,50.4$ (diene, $\mathrm{sp}^{2}-\mathrm{C}$ ), 31.5, 27.9, 19.6 (diene, $\left.\mathrm{sp}^{3}-\mathrm{C}\right), 10.0\left(\mathrm{Cp}^{-\mathrm{CH}_{3}}\right) \mathrm{ppm}$. Anal. Calc. (Found) for $\mathrm{C}_{17} \mathrm{H}_{26} \mathrm{ClRu}$ : $\mathrm{C}, 55.65$ (55.88); $\mathrm{H}, 7.14$ (7.35).

Cp*Ru(2-n-propylbutadiene)Cl. A procedure analogous to that employed for $\mathrm{Cp} * \mathrm{Ru}\left(2\right.$-ethylbutadiene)Cl, starting with $78.5 \mathrm{mg}(72.2 \mu \mathrm{mol})$ of $[\mathrm{Cp} * \mathrm{RuCl}]_{4}$ and 41.7 $\mathrm{mg}(0.433 \mathrm{mmol})$ of 2-propylbutadiene, led to the formation of the product as a yellow microcrystalline solid in $79 \%$ yield $(83.9 \mathrm{mg}, 0.228 \mathrm{mmol}) .{ }^{1} \mathrm{H}$ NMR $(500 \mathrm{MHz}$, $\left.\mathrm{CD}_{2} \mathrm{Cl}_{2}\right): \delta 4.41\left(\mathrm{dd}, 1 \mathrm{H},{ }^{3} J=10.2 \mathrm{~Hz},{ }^{3} J=7.6 \mathrm{~Hz},=\mathrm{C}-\mathrm{H}\right), 3.24\left(\mathrm{~s}, 1 \mathrm{H},=\mathrm{CH}_{2}\right), 3.09(\mathrm{dd}$, 
$\left.1 \mathrm{H},{ }^{2} J=1.5 \mathrm{~Hz},{ }^{3} J=7.5 \mathrm{~Hz},=\mathrm{CH}_{2}\right), 2.02-1.88\left(\mathrm{~m}, 2 \mathrm{H},-\mathrm{CH}_{2}-\right), 1.76-1.68\left(\mathrm{~m}, 2 \mathrm{H},-\mathrm{CH}_{2^{-}}\right)$, $1.61\left(\mathrm{~s}, 15 \mathrm{H}, \mathrm{Cp}-\mathrm{CH}_{3}\right), 1.36\left(\mathrm{~s}, 1 \mathrm{H},=\mathrm{CH}_{2}\right), 1.25\left(\mathrm{dd}, 1 \mathrm{H},{ }^{2} J=1.6 \mathrm{~Hz},{ }^{3} J=10.2 \mathrm{~Hz}\right.$, $\left.=\mathrm{CH}_{2}\right), 1.05\left(\mathrm{~d}, 3 \mathrm{H},{ }^{3} J=7.4 \mathrm{~Hz},-\mathrm{CH}_{3}\right) \mathrm{ppm} .{ }^{13} \mathrm{C}\left\{{ }^{1} \mathrm{H}\right\} \mathrm{NMR}\left(125 \mathrm{MHz}, \mathrm{CD}_{2} \mathrm{Cl}_{2}\right): 112.1$ (=-ㅡ-C-H), $95.6\left(\underline{\mathrm{p} p}-\mathrm{CH}_{3}\right), 90.1(=\mathrm{C}-\underline{\mathrm{C}}-\mathrm{H}), 53.4\left(=\mathrm{CH}_{2}\right), 53.4\left(=\mathrm{CH}_{2}\right), 38.8\left(-\mathrm{CH}_{2}-\right), 27.6$ $\left(-\mathrm{CH}_{2}-\right), 14.1\left(-\mathrm{CH}_{3}\right), 9.8\left(\mathrm{Cp}-\underline{\mathrm{CH}}_{3}\right)$ ppm. Anal. Calc. (Found) for $\mathrm{C}_{17} \mathrm{H}_{26} \mathrm{ClRu}: \mathrm{C}, 55.65$ (55.91); H, 7.14 (7.22).

Cp*Ru(1-ethylbutadiene)Cl. A procedure analogous to that employed for $\mathrm{Cp} * \mathrm{Ru}(2$-ethylbutadiene $) \mathrm{Cl}$, starting with $80.5 \mathrm{mg}(74.0 \mu \mathrm{mol})$ of $[\mathrm{Cp} * \mathrm{RuCl}]_{4}$ and 300 $\mu \mathrm{L}$ of 1-ethylbutadiene led to the formation of the product as an orange powder in $84 \%$ yield $(86.0 \mathrm{mg}, 0.243 \mathrm{mmol}) .{ }^{1} \mathrm{H}$ NMR (500 MHz, $d^{8}$-toluene): $\delta 4.05$ (dd, $1 \mathrm{H},{ }^{3} J=5.4$ $\left.\mathrm{Hz},{ }^{3} J=10.2 \mathrm{~Hz},=\mathrm{C}-\mathrm{H}\right), 3.76\left(\mathrm{dddd}, 1 \mathrm{H},{ }^{3} J=10.4 \mathrm{~Hz},{ }^{3} J=7.5 \mathrm{~Hz},{ }^{3} J=5.4 \mathrm{~Hz},{ }^{4} J=0.8\right.$ $\mathrm{Hz},=\mathrm{C}-\mathrm{H}), 2.87\left(\mathrm{ddd}, 1 \mathrm{H},{ }^{3} J=7.5 \mathrm{~Hz},{ }^{2} J=1.7 \mathrm{~Hz},{ }^{4} J=0.5 \mathrm{~Hz},=\mathrm{CH}_{2}\right), 2.38\left(\mathrm{dt}, 1 \mathrm{H},{ }^{3} J=\right.$ $\left.10.2 \mathrm{~Hz},{ }^{3} J=7.2 \mathrm{~Hz},=\mathrm{C}-\mathrm{H}\right), 1.81-1.65\left(\mathrm{~m}, 2 \mathrm{H},-\mathrm{CH}_{2}-\right), 1.69\left(\mathrm{ddd}, 1 \mathrm{H},{ }^{3} J=10.4 \mathrm{~Hz},{ }^{2} J\right.$ $\left.=1.9 \mathrm{~Hz},{ }^{4} \mathrm{~J}=0.8 \mathrm{~Hz},=\mathrm{CH}_{2}\right), 1.32\left(\mathrm{~s}, 15 \mathrm{H}, \mathrm{Cp}-\mathrm{CH}_{3}\right), 1.12\left(\mathrm{t}, 3 \mathrm{H},{ }^{3} \mathrm{~J}=7.4 \mathrm{~Hz},-\mathrm{CH}_{3}\right)$ ppm. ${ }^{13} \mathrm{C}\left\{{ }^{1} \mathrm{H}\right\}$ NMR (125 MHz, $d^{8}$-toluene): $94.0\left(\underline{\mathrm{Cp}}-\mathrm{CH}_{3}\right), 93.2,87.5,78.7,52.4$ (diene, sp ${ }^{2}-\mathrm{C}$ ), 26.1, 16.1 (diene, sp $\left.{ }^{3}-\mathrm{C}\right), 9.7\left(\mathrm{Cp}-\underline{\mathrm{CH}}_{3}\right) \mathrm{ppm}$. Anal. Calc. (Found) for $\mathrm{C}_{16} \mathrm{H}_{24} \mathrm{ClRu}: \mathrm{C}, 54.46$ (54.56); H, 6.86 (6.59).

Cp*Ru(1-n-propylbutadiene)Cl. A procedure analogous to that employed for $\mathrm{Cp} * \mathrm{Ru}(2$-ethylbutadiene $) \mathrm{Cl}$, starting with $[\mathrm{Cp} * \mathrm{RuCl}]_{4}(74.2 \mathrm{mg}, 68.3 \mu \mathrm{mol})$ and 1- $n$ propylbutadiene $(300 \mu \mathrm{L})$ the product was obtained in $90 \%$ yield $(90.4 \mathrm{mg}, 0.246 \mathrm{mmol})$. ${ }^{1} \mathrm{H}$ NMR (500 MHz, $d^{8}$-toluene): $\delta 4.10\left(\mathrm{dd}, 1 \mathrm{H},{ }^{3} J=10.3 \mathrm{~Hz},{ }^{3} J=5.3 \mathrm{~Hz},=\mathrm{C}-\mathrm{H}\right), 3.76$ 
(dddd, $\left.1 \mathrm{H},{ }^{3} J=10.5 \mathrm{~Hz},{ }^{3} J=7.5 \mathrm{~Hz},{ }^{3} J=5.4 \mathrm{~Hz},{ }^{4} J=0.8 \mathrm{~Hz},=\mathrm{C}-\mathrm{H}\right), 2.88\left(\mathrm{ddd}, 1 \mathrm{H},{ }^{3} J=\right.$ $\left.7.5 \mathrm{~Hz},{ }^{2} J=2.0 \mathrm{~Hz},{ }^{4} J=0.6 \mathrm{~Hz},=\mathrm{CH}_{2}\right), 2.40\left(\mathrm{ddt}, 1 \mathrm{H},{ }^{3} J=10.3 \mathrm{~Hz},{ }^{3} J=6.8 \mathrm{~Hz},{ }^{4} J=0.8\right.$ $\mathrm{Hz},=\mathrm{C}-\mathrm{H}), 1.83\left(\mathrm{~m}, 1 \mathrm{H},-\mathrm{CH}_{2^{-}}\right), 1.70\left(\mathrm{ddd}, 1 \mathrm{H},{ }^{3} J=10.5 \mathrm{~Hz},{ }^{2} J=2.0 \mathrm{~Hz},{ }^{4} J=0.8 \mathrm{~Hz}\right.$, $\left.=\mathrm{CH}_{2}\right), 1.64-1.49\left(\mathrm{~m}, 3 \mathrm{H},-\mathrm{CH}_{2}-\right), 1.33\left(\mathrm{~s}, 15 \mathrm{H}, \mathrm{Cp}-\mathrm{CH}_{3}\right), 0.97\left(\mathrm{t}, 3 \mathrm{H},{ }^{3} \mathrm{~J}=7.4 \mathrm{~Hz}\right.$, $\left.\mathrm{CH}_{3}\right)$ ppm. ${ }^{13} \mathrm{C}\left\{{ }^{1} \mathrm{H}\right\}$ NMR (125 MHz, $d^{8}$-toluene): $94.0\left(\underline{\mathrm{Cp}}-\mathrm{CH}_{3}\right), 93.7,87.5,76.7,52.4$ (diene, $\mathrm{sp}^{2}-\mathrm{C}$ ), 35.1, 24.8, 14.2 (diene, $\mathrm{sp}^{3}-\mathrm{C}$ ), $9.2\left(\mathrm{Cp}^{-} \mathrm{CH}_{3}\right) \mathrm{ppm}$. Anal. Calc. (Found) for $\mathrm{C}_{17} \mathrm{H}_{26} \mathrm{ClRu}: \mathrm{C}, 55.65$ (55.80); H, 7.14 (7.42).

\section{$\underline{\text { Host-guest complexes }}$}

General remarks: Assignments of diastereomers $A$ and $B$ ( $A$ being the dominant diastereomer if applicable) were made based on TOCSY and NOESY spectra or integration where possible. The coordinated water molecule in the host-guest complexes was not observed by ${ }^{1} \mathrm{H}$ NMR, presumably due to fast chemical exchange with the NMR solvent $\mathrm{D}_{2} \mathrm{O}$. Also attempts to observe the host-guest complexes by ES(-) mass spectrometry remained unsuccessful. However, the analogous "solvated" acetonitrile complexes $\mathrm{K}_{5}\left(\mathrm{NMe}_{4}\right)_{6}\left[\mathrm{CpRu}\left(2,3\right.\right.$-dimethylbutadiene)(MeCN) $\left.\subset \mathrm{Ga}_{4} \mathrm{~L}_{6}\right] \quad$ (2b) and $\mathrm{K}_{11}\left[\mathrm{Cp} * \mathrm{Ru}(\right.$ isoprene $\left.)(\mathrm{MeCN}) \subset \mathrm{Ga}_{4} \mathrm{~L}_{6}\right](\mathbf{5 b})$ confirmed their composition in the mass spectrum as stated. Due to lower binding constants for the $\mathrm{Cp} * \mathrm{Ru}$-complexes, complete exchange of $\mathrm{NMe}_{4}{ }^{+}$for these larger guest molecules is not possible. We therefore explored encapsulation experiments with the empty assembly $\mathrm{K}_{12}\left[\mathrm{Ga}_{4} \mathrm{~L}_{6}\right]$ (1) $)$. The resolution experiment was conducted in the presence of 1 eq. $\mathrm{NMe}_{4}{ }^{+}$to prevent racemization of the empty resolved assembly. 
General encapsulation protocol: Under a nitrogen atmosphere an aqueous solution of $\mathbf{1 a}$ or $\mathbf{1 b}$ was added to an ethereal solution of Ru-complex in equimolar concentration. Vigorous stirring at room temperature for $2 \mathrm{~h}$ (30 min for the $\mathrm{Cp}$-complexes) led to loss of the yellow/orange color of the organic layer, indicating that all of the Ru-complex had dissolved in the aqueous layer. The two layers were separated. The host-guest complexes were isolated from the aqueous layer after removal of $\mathrm{H}_{2} \mathrm{O}$ and placement in the vacuum oven at $45^{\circ} \mathrm{C}$ overnight as yellow solids in almost quantitative yield.

$\mathbf{K}_{5}\left(\mathrm{NMe}_{4}\right)_{6}\left[\mathbf{C p R u}(\mathbf{2 , 3 - d i m e t h y l b u t a d i e n e})\left(\mathbf{H}_{2} \mathrm{O}\right) \subset \mathbf{G a}_{4} \mathbf{L}_{6}\right](\mathbf{2 a}) .{ }^{1} \mathrm{H}$ NMR $(500$ $\left.\mathrm{MHz}, \mathrm{D}_{2} \mathrm{O}\right)$, N-H not observed due to H-D exchange: $\delta 7.91\left(\mathrm{~d},{ }^{3} J=7.8 \mathrm{~Hz}, 12 \mathrm{H}, \mathrm{Ar}-\mathrm{H}\right)$, $7.70\left(\mathrm{~d},{ }^{3} J=7.5 \mathrm{~Hz}, 12 \mathrm{H}, \mathrm{Ar}-\mathrm{H}\right), 7.15\left(\mathrm{~d},{ }^{3} J=8.1 \mathrm{~Hz}, 12 \mathrm{H}, \mathrm{Ar}-\mathrm{H}\right), 6.85\left(\mathrm{t},{ }^{3} J=8.4 \mathrm{~Hz}\right.$, 12H, Ar-H), 6.54 (s, br, 12H, Ar-H), $6.43\left(\mathrm{t},{ }^{3} \mathrm{~J}=7.8 \mathrm{~Hz}, 12 \mathrm{H}, \mathrm{Ar}-\mathrm{H}\right), 2.55$ (s, 72H, $\mathrm{NMe}_{4}$, exterior), 1.41 (s, 5H, Cp-H, encaps.), 1.07 (s, 1H, =CH $\mathrm{CH}_{2}$, encaps.), 0.68 (s, $1 \mathrm{H}$, $=\mathrm{CH}_{2}$, encaps.), -0.47 (s, $3 \mathrm{H}, \mathrm{CH}_{3}$, encaps.), -0.73 (s, $3 \mathrm{H}, \mathrm{CH}_{3}$, encaps.), -2.21 (s, $2 \mathrm{H}$, $=\mathrm{CH}_{2}$, encaps.) $\mathrm{ppm}$.

\section{$\mathrm{K}_{5}\left(\mathrm{NMe}_{4}\right)_{6}\left[\mathrm{CpRu}(2,3-\right.$ dimethylbutadiene $\left.)(\mathrm{MeCN}) \subset \mathbf{G a}_{4} \mathbf{L}_{6}\right](\mathbf{2 b}) .{ }^{1} \mathrm{H}$ NMR}

(500 MHz, $\mathrm{D}_{2} \mathrm{O}$ ), N-H not observed due to H-D exchange: $\delta 7.93\left(\mathrm{~d},{ }^{3} J=7.8 \mathrm{~Hz}, 12 \mathrm{H}\right.$, Ar-H), $7.72\left(\mathrm{~d},{ }^{3} J=8.5 \mathrm{~Hz}, 12 \mathrm{H}, \mathrm{Ar}-\mathrm{H}\right), 7.20\left(\mathrm{dd},{ }^{3} J=8.3 \mathrm{~Hz},{ }^{4} J=1.6 \mathrm{~Hz}, 12 \mathrm{H}, \mathrm{Ar}-\mathrm{H}\right)$, $6.94\left(\mathrm{t},{ }^{3} J=8.1 \mathrm{~Hz}, 12 \mathrm{H}, \mathrm{Ar}-\mathrm{H}\right), 6.64\left(\mathrm{dd},{ }^{3} J=7.4 \mathrm{~Hz},{ }^{2} J=1.6 \mathrm{~Hz}, 12 \mathrm{H}, \mathrm{Ar}-\mathrm{H}\right), 6.50\left(\mathrm{t},{ }^{3} J\right.$ $=7.8 \mathrm{~Hz}, 12 \mathrm{H}, \mathrm{Ar}-\mathrm{H}$ ), 2.54 (s, 72H, NMe 4 , exterior), 1.98 (s, 5H, Cp-H, encaps.), 0.78 (s, $1 \mathrm{H},=\mathrm{CH}_{2}$, encaps.), 0.74 (s, $3 \mathrm{H}, \mathrm{CH}_{3}$, encaps.), 0.68 (s, $1 \mathrm{H},=\mathrm{CH}_{2}$, encaps.), -0.17 (s, $3 \mathrm{H}$, $\mathrm{CH}_{3}$, encaps.), -1.03 (s, $3 \mathrm{H}, \mathrm{CH}_{3}$, encaps.), -2.07 (s, $1 \mathrm{H},=\mathrm{CH}_{2}$, encaps.), -2.38 (s, $1 \mathrm{H}$, 
$=\mathrm{CH}_{2}$, encaps. $)$ ppm. ES(-)-MS $\left(\mathrm{H}_{2} \mathrm{O} / \mathrm{MeOH}, 70: 30\right) \bullet=\left[\mathrm{Ga}_{4} \mathrm{~L}_{6}\right]^{12-}, \mathrm{m} / z: 640$ $\left[\mathrm{CpRu}(2,3-\text { dimethylbutadiene })(\mathrm{MeCN})^{+} \subset+3 \mathrm{Na}^{+}+3 \mathrm{H}^{+}\right]^{5-}, 644 \quad[\mathrm{CpRu}(2,3-$ dimethylbutadiene) $\left.(\mathrm{MeCN})^{+} \subset \bullet+4 \mathrm{Na}^{+}+2 \mathrm{H}^{+}\right]^{5-}, 649 \quad[\mathrm{CpRu}(2,3-$ dimethylbutadiene) $\left.(\mathrm{MeCN})^{+} \subset \bullet+5 \mathrm{Na}^{+}+1 \mathrm{H}^{+}\right]^{5-}, 653 \quad[\mathrm{CpRu}(2,3-$ dimethylbutadiene) $\left.(\mathrm{MeCN})^{+} \subset+6 \mathrm{Na}^{+}\right]^{5-}, 806\left[\mathrm{CpRu}(2,3\right.$-dimethylbutadiene $)(\mathrm{MeCN})^{+}$ $\left.\subset+4 \mathrm{Na}^{+}+3 \mathrm{H}^{+}\right]^{4-}, 811\left[\mathrm{CpRu}(2,3-\text { dimethylbutadiene })(\mathrm{MeCN})^{+} \subset+5 \mathrm{Na}^{+}+2 \mathrm{H}^{+}\right]^{4-}$, $817\left[\mathrm{CpRu}(2,3-\text { dimethylbutadiene })(\mathrm{MeCN})^{+} \subset+6 \mathrm{Na}^{+}+1 \mathrm{H}^{+}\right]^{4-}, 822 \quad[\mathrm{CpRu}(2,3-$ dimethylbutadiene $\left.)(\mathrm{MeCN})^{+} \subset+7 \mathrm{Na}^{+}\right]^{4-}$.

$\mathbf{K}_{5}\left(\mathbf{N M e}_{4}\right)_{6}\left[\mathbf{C p R u}(\right.$ isoprene $\left.)\left(\mathbf{H}_{2} \mathbf{O}\right) \subset \mathbf{G a}_{4} \mathbf{L}_{6}\right](3) .{ }^{1} \mathrm{H}$ NMR $\left(500 \mathrm{MHz}, \mathrm{D}_{2} \mathrm{O}\right)$, N-H not observed due to H-D exchange: $\delta 7.93\left(\mathrm{~d},{ }^{3} J=7.9 \mathrm{~Hz}, 12 \mathrm{H}, \mathrm{Ar}-\mathrm{H}\right), 7.91\left(\mathrm{~d},{ }^{3} J=\right.$ $7.9 \mathrm{~Hz}, 12 \mathrm{H}, \mathrm{Ar}-\mathrm{H}), 7.66\left(\mathrm{~d},{ }^{3} \mathrm{~J}=7.7 \mathrm{~Hz}, 12 \mathrm{H}, \mathrm{Ar}-\mathrm{H}\right), 7.65\left(\mathrm{~d},{ }^{3} J=7.4 \mathrm{~Hz}, 12 \mathrm{H}, \mathrm{Ar}-\mathrm{H}\right)$, $7.21\left(\mathrm{dd},{ }^{3} J=8.3 \mathrm{~Hz},{ }^{4} J=1.5 \mathrm{~Hz}, 12 \mathrm{H}, \mathrm{Ar}-\mathrm{H}\right), 7.20\left(\mathrm{dd},{ }^{3} J=8.3 \mathrm{~Hz},{ }^{4} J=1.5 \mathrm{~Hz}, 12 \mathrm{H}\right.$, Ar-H), $6.93\left(\mathrm{t},{ }^{3} J=8.5 \mathrm{~Hz}, 12 \mathrm{H}, \mathrm{Ar}-\mathrm{H}\right), 6.91\left(\mathrm{t},{ }^{3} \mathrm{~J}=8.6 \mathrm{~Hz}, 12 \mathrm{H}, \mathrm{Ar}-\mathrm{H}\right), 6.61\left(\mathrm{~d},{ }^{3} J=7.4\right.$ $\mathrm{Hz}, 24 \mathrm{H}, \mathrm{Ar}-\mathrm{H}$ ), 6.47 (t, $\left.{ }^{3} \mathrm{~J}=8.0 \mathrm{~Hz}, 24 \mathrm{H}, \mathrm{Ar}-\mathrm{H}\right), 2.53$ (s, 144H, $\mathrm{NMe}_{4}$, exterior), 1.71 (dd, ${ }^{3} J=11.0 \mathrm{~Hz},{ }^{3} J=8.2 \mathrm{~Hz}, 1 \mathrm{H},=\mathrm{C}-\mathrm{H}$, encaps. $A$ ), 1.62 (s, $5 \mathrm{H}, \mathrm{Cp}-\mathrm{H}$, encaps.), 1.45 (s, 5H, Cp-H, encaps.), 1.42 (dd, ${ }^{3} J=8.1 \mathrm{~Hz}, 1 \mathrm{H},=\mathrm{C}-\mathrm{H}$, encaps. $\left.B\right), 1.36\left(\mathrm{~d},{ }^{3} J=7.9 \mathrm{~Hz}\right.$, $=\mathrm{CH}_{2}$, encaps. $\left.B\right), 1.01\left(\mathrm{~d},{ }^{3} J=8.4 \mathrm{~Hz},=\mathrm{CH}_{2}\right.$, encaps. $\left.A\right), 0.92\left(\mathrm{~s}, 1 \mathrm{H},=\mathrm{CH}_{2}\right.$, encaps. $\left.B\right)$, $0.88\left(\mathrm{~s}, 1 \mathrm{H},=\mathrm{CH}_{2}\right.$, encaps. $\left.A\right),-0.10$ (s, $3 \mathrm{H}, \mathrm{CH}_{3}$, encaps. $\left.A\right),-0.21$ (s, $3 \mathrm{H}, \mathrm{CH}_{3}$, encaps. $B),-1.95\left(\mathrm{~d},{ }^{3} \mathrm{~J}=10.8 \mathrm{~Hz}, 1 \mathrm{H},=\mathrm{CH}_{2}\right.$, encaps. $\left.B\right),-1.96\left(\mathrm{~d},{ }^{3} J=11.4 \mathrm{~Hz}, 1 \mathrm{H},=\mathrm{CH}_{2}\right.$, encaps. $A$ ),-2.39 (s, $1 \mathrm{H},=\mathrm{CH}_{2}$, encaps. $\left.B\right),-2.55$ (s, $1 \mathrm{H},=\mathrm{CH}_{2}$, encaps. $A$ ) ppm. TOCSY and NOESY spectra were also recorded. 
$\mathbf{K}_{5}\left(\mathbf{N M e}_{4}\right)_{6}\left[\mathbf{C p R u}(2\right.$-ethylbutadiene $\left.)\left(\mathbf{H}_{2} \mathbf{O}\right) \subset \mathbf{G a}_{4} \mathbf{L}_{6}\right](\mathbf{4}) .{ }^{1} \mathrm{H}$ NMR $(500 \mathrm{MHz}$, $\mathrm{D}_{2} \mathrm{O}$ ), N-H not observed due to H-D exchange: $\delta 7.94\left(\mathrm{~d},{ }^{3} J=7.9 \mathrm{~Hz}, 12 \mathrm{H}, \mathrm{Ar}-\mathrm{H}\right), 7.92$ $\left(\mathrm{d},{ }^{3} J=7.9 \mathrm{~Hz}, 12 \mathrm{H}, \mathrm{Ar}-\mathrm{H}\right), 7.71\left(\mathrm{~d},{ }^{3} J=7.7 \mathrm{~Hz}, 12 \mathrm{H}, \mathrm{Ar}-\mathrm{H}\right), 7.70\left(\mathrm{~d},{ }^{3} J=7.4 \mathrm{~Hz}, 12 \mathrm{H}\right.$, Ar-H), $7.17\left(\mathrm{~d},{ }^{3} J=8.3 \mathrm{~Hz}, 24 \mathrm{H}, \mathrm{Ar}-\mathrm{H}\right), 6.88\left(\mathrm{t},{ }^{3} J=8.5 \mathrm{~Hz}, 12 \mathrm{H}, \mathrm{Ar}-\mathrm{H}\right), 6.86\left(\mathrm{t},{ }^{3} J=8.6\right.$ $\mathrm{Hz}, 12 \mathrm{H}, \mathrm{Ar}-\mathrm{H}), 6.59$ (d, $\left.{ }^{3} J=7.4 \mathrm{~Hz}, 24 \mathrm{H}, \mathrm{Ar}-\mathrm{H}\right), 6.45\left(\mathrm{t},{ }^{3} \mathrm{~J}=8.0 \mathrm{~Hz}, 24 \mathrm{H}, \mathrm{Ar}-\mathrm{H}\right), 2.47$ (s, 144H, $\mathrm{NMe}_{4}$, exterior), 2.08 (s, 5H, Cp-H, encaps.), 2.00 (s, 5H, Cp-H, encaps.), 1.93 $\left(\mathrm{dd},{ }^{3} \mathrm{~J}=10.0 \mathrm{~Hz},{ }^{3} J=8.6 \mathrm{~Hz}, 1 \mathrm{H},=\mathrm{C}-\mathrm{H}\right.$, encaps. $\left.A\right), 1.81\left(\mathrm{~d},{ }^{3} J=7.2 \mathrm{~Hz}, 1 \mathrm{H},=\mathrm{CH}_{2}\right.$, encaps. $B), 1.67\left(\mathrm{~d},{ }^{3} J=7.0 \mathrm{~Hz}, 1 \mathrm{H},=\mathrm{CH}_{2}\right.$, encaps. $\left.A\right), 1.52\left(\mathrm{dd},{ }^{3} J=10.2 \mathrm{~Hz},{ }^{3} J=8.8\right.$ $\mathrm{Hz}, 1 \mathrm{H},=\mathrm{C}-\mathrm{H}$, encaps. $B), 1.15\left(\mathrm{~s}, 1 \mathrm{H},=\mathrm{CH}_{2}\right.$, encaps. $\left.B\right), 1.04\left(\mathrm{~s}, 1 \mathrm{H},=\mathrm{CH}_{2}\right.$, encaps. $\left.A\right)$, 0.63 (t, ${ }^{3} J=7.4 \mathrm{~Hz}, 3 \mathrm{H}, \mathrm{CH}_{3}$, encaps.), -0.71 (t, ${ }^{3} J=7.4 \mathrm{~Hz}, 3 \mathrm{H}, \mathrm{CH}_{3}$, encaps.), -1.58 (m, $2 \mathrm{H}, \mathrm{CH}_{2}$, encaps.), $-1.74\left(\mathrm{~d},{ }^{3} \mathrm{~J}=11.0 \mathrm{~Hz}, 1 \mathrm{H},=\mathrm{CH}_{2}\right.$, encaps. $\left.B\right),-1.83\left(\mathrm{~d},{ }^{3} \mathrm{~J}=10.8 \mathrm{~Hz}\right.$, $1 \mathrm{H},=\mathrm{CH}_{2}$, encaps. $A$ ), -1.93 (m, $2 \mathrm{H}, \mathrm{CH}_{2}$, encaps.), -2.22 (s, $1 \mathrm{H},=\mathrm{CH}_{2}$, encaps. $\left.A\right),-2.49$ $\left(\mathrm{s}, 1 \mathrm{H},=\mathrm{CH}_{2}\right.$, encaps. $\left.B\right)$ ppm. TOCSY and NOESY spectra were also recorded.

$\mathbf{K}_{11}\left[\mathbf{C} \mathbf{p} * \mathbf{R u}(\right.$ isoprene $\left.)\left(\mathbf{H}_{2} \mathbf{O}\right) \subset \mathbf{G a}_{4} \mathbf{L}_{6}\right](\mathbf{5 a}) .{ }^{1} \mathrm{H}$ NMR $\left(500 \mathrm{MHz}, \mathrm{D}_{2} \mathrm{O}\right), \mathrm{N}-\mathrm{H}$ not observed due to H-D exchange, aromatic region broad: $\delta 1.34\left(\mathrm{dd},{ }^{3} J=9.5 \mathrm{~Hz},{ }^{3} J=8.5\right.$ $\mathrm{Hz}, 1 \mathrm{H},=\mathrm{C}-\mathrm{H}$, encaps.), 1.05 (dd, ${ }^{3} J=9.0 \mathrm{~Hz},{ }^{3} \mathrm{~J}=8.9 \mathrm{~Hz}, 1 \mathrm{H},=\mathrm{C}-\mathrm{H}$, encaps.), 0.79 (s, $1 \mathrm{H},=\mathrm{CH}_{2}$, encaps.), 0.67 (s, $1 \mathrm{H},=\mathrm{CH}_{2}$, encaps.), $0.48\left(\mathrm{~d},{ }^{3} \mathrm{~J}=7.0 \mathrm{~Hz}, 1 \mathrm{H},=\mathrm{CH}_{2}\right.$, encaps.), 0.39 (s, $3 \mathrm{H}, \mathrm{CH}_{3}$, encaps.), 0.35 (d, ${ }^{3} \mathrm{~J}=6.8 \mathrm{~Hz}, 1 \mathrm{H},=\mathrm{CH}_{2}$, encaps.), 0.32 (s, 3H, $\mathrm{CH}_{3}$, encaps.), -0.94 (s, 15H, $\mathrm{Cp}-\mathrm{CH}_{3}$, encaps.), -0.99 (s, $15 \mathrm{H}, \mathrm{Cp}-\mathrm{CH}_{3}$, encaps.), $2.04\left(\mathrm{~d},{ }^{3} \mathrm{~J}=10.4 \mathrm{~Hz}, 1 \mathrm{H},=\mathrm{CH}_{2}\right.$, encaps.), $-2.38\left(\mathrm{~d},{ }^{3} \mathrm{~J}=10.2 \mathrm{~Hz}, 1 \mathrm{H},=\mathrm{CH}_{2}\right.$, encaps. $)$ 2.67 (s, $1 \mathrm{H},=\mathrm{CH}_{2}$, encaps.), -2.69 (s, $1 \mathrm{H},=\mathrm{CH}_{2}$, encaps.) ppm. 
$\mathbf{K}_{11}\left[\mathbf{C p} * \mathbf{R u}(\right.$ isoprene $\left.)(\mathbf{M e C N}) \subset \mathbf{G a}_{4} \mathbf{L}_{6}\right](\mathbf{5 b}) .{ }^{1} \mathrm{H}$ NMR $\left(500 \mathrm{MHz}, \mathrm{D}_{2} \mathrm{O}\right), \mathrm{N}-\mathrm{H}$ not observed due to $\mathrm{H}-\mathrm{D}$ exchange, aromatic region broad: $\delta 2.37$ (s, $3 \mathrm{H}, \mathrm{CH}_{3}$, encaps.), 2.11 (s, 3H, $\mathrm{CH}_{3}$, encaps.), 0.80 (dd, ${ }^{3} J=10.0 \mathrm{~Hz},{ }^{3} J=8.2 \mathrm{~Hz}, 1 \mathrm{H},=\mathrm{C}-\mathrm{H}$, encaps.), 0.47 (dd, ${ }^{3} J=9.6 \mathrm{~Hz},{ }^{3} J=7.8 \mathrm{~Hz}, 1 \mathrm{H},=\mathrm{C}-\mathrm{H}$, encaps.), 0.39 (s, $1 \mathrm{H},=\mathrm{CH}_{2}$, encaps.), 0.37 (d, ${ }^{3} J$ $=7.2 \mathrm{~Hz}, 1 \mathrm{H},=\mathrm{CH}_{2}$, encaps.), 0.34 (s, $3 \mathrm{H}, \mathrm{CH}_{3}$, encaps.), 0.29 (s, $3 \mathrm{H}, \mathrm{CH}_{3}$, encaps.), 0.11 (s, $1 \mathrm{H},=\mathrm{CH}_{2}$, encaps.), -0.65 (s, $15 \mathrm{H}, \mathrm{Cp}-\mathrm{CH}_{3}$, encaps.), -0.67 (s, $15 \mathrm{H}, \mathrm{Cp}-\mathrm{CH}_{3}$, encaps.), $-2.30\left(\mathrm{~d},{ }^{3} J=10.0 \mathrm{~Hz}, 1 \mathrm{H},=\mathrm{CH}_{2}\right.$, encaps. $),-2.36\left(\mathrm{~d},{ }^{3} J=10.1 \mathrm{~Hz}, 1 \mathrm{H},=\mathrm{CH}_{2}\right.$, encaps. $),-$ 2.78 (s, $1 \mathrm{H},=\mathrm{CH}_{2}$, encaps.), -2.90 (s, $1 \mathrm{H},=\mathrm{CH}_{2}$, encaps.) ppm. $\mathrm{ES}(-)-\mathrm{MS}\left(\mathrm{H}_{2} \mathrm{O} / \mathrm{MeOH}\right.$, $70: 30) \bullet=\left[\mathrm{Ga}_{4} \mathrm{~L}_{6}\right]^{12-}, m / z: 646\left[\mathrm{Cp} * \mathrm{Ru}(\text { isoprene })(\mathrm{MeCN})^{+} \subset+2 \mathrm{Na}^{+}+4 \mathrm{H}^{+}\right]^{5-}, 651$ $\left[\mathrm{Cp} * \mathrm{Ru}(\text { isoprene })(\mathrm{MeCN})^{+} \subset+3 \mathrm{Na}^{+}+3 \mathrm{H}^{+}\right]^{5-}, 655\left[\mathrm{Cp} * \mathrm{Ru}(\right.$ isoprene $)(\mathrm{MeCN})^{+} \subset+$ $\left.4 \mathrm{Na}^{+}+2 \mathrm{H}^{+}\right]^{5-}, 660\left[\mathrm{Cp} * \mathrm{Ru}(\text { isoprene })(\mathrm{MeCN})^{+} \subset++5 \mathrm{Na}^{+}+1 \mathrm{H}^{+}\right]^{5-}, 664$ $\left[\mathrm{Cp} * \mathrm{Ru}(\text { isoprene })(\mathrm{MeCN})^{+} \subset+6 \mathrm{Na}^{+}\right]^{5-}, 820\left[\mathrm{Cp} * \mathrm{Ru}(\right.$ isoprene $)(\mathrm{MeCN})^{+} \subset+4 \mathrm{Na}^{+}+$ $\left.3 \mathrm{H}^{+}\right]^{4-}, 825 \quad\left[\mathrm{Cp} * \mathrm{Ru}\left(\text { isoprene) }(\mathrm{MeCN})^{+} \subset++5 \mathrm{Na}^{+}+2 \mathrm{H}^{+}\right]^{4-}, 831\right.$ $\left[\mathrm{Cp} * \mathrm{Ru}(\text { isoprene })(\mathrm{MeCN})^{+} \subset+6 \mathrm{Na}^{+}+1 \mathrm{H}^{+}\right]^{4-}, 836\left[\mathrm{Cp} * \mathrm{Ru}(\right.$ isoprene $)(\mathrm{MeCN})^{+} \subset+$ $\left.7 \mathrm{Na}^{+}\right]^{4-}$.

$\mathbf{K}_{11}\left[\mathbf{C p} * \mathbf{R u}\left(\mathbf{2}\right.\right.$-ethylbutadiene) $\left.\left(\mathbf{H}_{2} \mathbf{O}\right) \subset \mathbf{G a}_{4} \mathbf{L}_{6}\right](\mathbf{6}) .{ }^{1} \mathrm{H} \mathrm{NMR}\left(500 \mathrm{MHz}, \mathrm{D}_{2} \mathrm{O}\right)$, N-H not observed due to H-D exchange, aromatic region broad: $\delta 1.40\left(\mathrm{dd},{ }^{3} J=9.1 \mathrm{~Hz}\right.$, ${ }^{3} J=8.3 \mathrm{~Hz}, 1 \mathrm{H},=\mathrm{C}-\mathrm{H}$, encaps. $\left.A\right), 1.15(\mathrm{dd}, 1 \mathrm{H},=\mathrm{C}-\mathrm{H}$, encaps. $B), 0.73\left(\mathrm{~d},{ }^{3} J=7.0 \mathrm{~Hz}\right.$, $1 \mathrm{H},=\mathrm{CH}_{2}$, encaps. $\left.A\right), 0.57$ (t, br, $3 \mathrm{H}, \mathrm{CH}_{3}$, encaps. $\left.B\right), 0.52\left(\mathrm{~s}, 1 \mathrm{H},=\mathrm{CH}_{2}\right.$, encaps. $\left.A\right)$, $0.34\left(\mathrm{~s}, 1 \mathrm{H},=\mathrm{CH}_{2}\right.$, encaps. B), $0.26\left(\mathrm{t}, \mathrm{br}, 3 \mathrm{H}, \mathrm{CH}_{3}\right.$, encaps. $\left.A\right), 0.24\left(\mathrm{~d}, 1 \mathrm{H},=\mathrm{CH}_{2}\right.$, 
encaps. $B),-0.69\left(\mathrm{~s}, 15 \mathrm{H}, \mathrm{Cp}-\mathrm{CH}_{3}\right.$, encaps. $\left.B\right),-0.89$ (s, $15 \mathrm{H}, \mathrm{Cp}-\mathrm{CH}_{3}$, encaps. $\left.A\right),-1.19$ $\left(\mathrm{m}, 1 \mathrm{H},-\mathrm{CH}_{2^{-}}\right.$, encaps. $\left.A\right),-1.23\left(\mathrm{~m}, 2 \mathrm{H},-\mathrm{CH}_{2^{-}}\right.$, encaps. $\left.B\right),-1.42\left(\mathrm{~m}, 1 \mathrm{H},-\mathrm{CH}_{2^{-}}\right.$, encaps. A), $-1.54\left(\mathrm{~d}, 1 \mathrm{H},=\mathrm{CH}_{2}\right.$, encaps. $\left.B\right),-1.95\left(\mathrm{~d},{ }^{3} J=10.1 \mathrm{~Hz}, 1 \mathrm{H},=\mathrm{CH}_{2}\right.$, encaps. $\left.A\right),-2.38$ (s, $1 \mathrm{H},=\mathrm{CH}_{2}$, encaps. $\left.A\right),-2.55\left(\mathrm{~s}, 1 \mathrm{H},=\mathrm{CH}_{2}\right.$, encaps. $\left.B\right)$ ppm.

$\mathbf{K}_{11}\left[\mathbf{C p} * \mathbf{R u}\left(2\right.\right.$-isopropylbutadiene) $\left.\left(\mathbf{H}_{2} \mathbf{O}\right) \subset \mathbf{G a}_{4} \mathbf{L}_{6}\right]$ (7). ${ }^{1} \mathrm{H}$ NMR $(500 \mathrm{MHz}$, $\left.\mathrm{D}_{2} \mathrm{O}\right), \mathrm{N}-\mathrm{H}$ not observed due to H-D exchange, aromatic region broad: $\delta 1.56\left(\mathrm{dd},{ }^{3} \mathrm{~J}=\right.$ $9.0 \mathrm{~Hz},{ }^{3} J=8.3 \mathrm{~Hz}, 1 \mathrm{H},=\mathrm{C}-\mathrm{H}$, encaps. $B$ ), 1.16 (s, $1 \mathrm{H},=\mathrm{CH}_{2}$, encaps. $B$ ), $1.05\left(\mathrm{dd},{ }^{3} J=\right.$ $9.2 \mathrm{~Hz},{ }^{3} J=8.0 \mathrm{~Hz}, 1 \mathrm{H},=\mathrm{C}-\mathrm{H}$, encaps. $\left.A\right), 0.88\left(\mathrm{~s}, 1 \mathrm{H},=\mathrm{CH}_{2}\right.$, encaps. $\left.A\right), 0.60\left(\mathrm{~d},{ }^{3} J=\right.$ $7.5 \mathrm{~Hz}, 1 \mathrm{H},=\mathrm{CH}_{2}$, encaps. $B$ ), $0.44\left(\mathrm{~d},{ }^{3} J=7.1 \mathrm{~Hz}, 3 \mathrm{H}, i\right.$-Pr $\mathrm{CH}_{3}$, encaps. $\left.A\right), 0.28\left(\mathrm{~d},{ }^{3} J=\right.$ 7.6 Hz, $1 \mathrm{H},=\mathrm{CH}_{2}$, encaps. A), $0.16\left(\mathrm{~d},{ }^{3} J=6.8 \mathrm{~Hz}, 3 \mathrm{H}, i-\mathrm{Pr} \mathrm{CH}_{3}\right.$, encaps. $\left.B\right),-0.58$ (s, $15 \mathrm{H}, \mathrm{Cp}-\mathrm{CH}_{3}$, encaps. $A$ ), -0.71 (s, $15 \mathrm{H}, \mathrm{Cp}-\mathrm{CH}_{3}$, encaps. $B$ ), -1.08 (m, $1 \mathrm{H}, i-\mathrm{Pr} \mathrm{C}-\mathrm{H}$, encaps. B), -1.29 (m, $1 \mathrm{H}, i$-Pr C-H, encaps. A), $-1.32\left(\mathrm{~d},{ }^{3} \mathrm{~J}=6.5 \mathrm{~Hz}, 3 \mathrm{H}, i-\mathrm{Pr} \mathrm{CH}_{3}\right.$, encaps. $B),-1.48\left(\mathrm{~d},{ }^{3} J=9.5 \mathrm{~Hz}, 1 \mathrm{H},=\mathrm{CH}_{2}\right.$, encaps. $\left.B\right),-1.66\left(\mathrm{~d},{ }^{3} J=6.6 \mathrm{~Hz}, 3 \mathrm{H}, i-\mathrm{Pr}\right.$ $\mathrm{CH}_{3}$, encaps. A), $-1.90\left(\mathrm{~d},{ }^{3} \mathrm{~J}=9.4 \mathrm{~Hz}, 1 \mathrm{H},=\mathrm{CH}_{2}\right.$, encaps. $\left.A\right),-2.17$ (s, $1 \mathrm{H},=\mathrm{CH}_{2}$, encaps. A), $-2.39\left(\mathrm{~s}, 1 \mathrm{H},=\mathrm{CH}_{2}\right.$, encaps. $\left.B\right)$ ppm.

\section{$\mathbf{K}_{11}\left[\mathbf{C p} * \mathbf{R u}(\mathbf{2}-\boldsymbol{n}\right.$-propylbutadiene $\left.)\left(\mathbf{H}_{\mathbf{2}} \mathbf{O}\right) \subset \mathbf{G a}_{4} \mathbf{L}_{6}\right]$ (8). ${ }^{1} \mathrm{H}$ NMR $(500 \mathrm{MHz}$,}

$\mathrm{D}_{2} \mathrm{O}$ ), N-H not observed due to $\mathrm{H}-\mathrm{D}$ exchange, aromatic region broad, major diastereomer: $\delta 1.25$ (dd, ${ }^{3} J=9.6 \mathrm{~Hz},{ }^{3} J=7.3 \mathrm{~Hz}, 1 \mathrm{H},=\mathrm{C}-\mathrm{H}$, encaps.), $0.74\left(\mathrm{~d},{ }^{3} J=7.0\right.$ $\mathrm{Hz}, 1 \mathrm{H},=\mathrm{CH}_{2}$, encaps.), 0.60 (s, $1 \mathrm{H},=\mathrm{CH}_{2}$, encaps.), -0.45 (t, br, $3 \mathrm{H}, \mathrm{CH}_{3}$, encaps.), $0.62\left(\mathrm{~m}, 1 \mathrm{H},-\mathrm{CH}_{2^{-}}\right.$, encaps.), -0.74 (s, $15 \mathrm{H}, \mathrm{Cp}-\mathrm{CH}_{3}$, encaps.), $-1.04\left(\mathrm{~m}, 2 \mathrm{H},-\mathrm{CH}_{2^{-}}\right.$, 
encaps.), -1.21 (m, $1 \mathrm{H},-\mathrm{CH}_{2-}$, encaps.), $-1.61\left(\mathrm{~d},{ }^{3} J=10.0 \mathrm{~Hz}, 1 \mathrm{H},=\mathrm{CH}_{2}\right.$, encaps.), -1.71 (s, $1 \mathrm{H},=\mathrm{CH}_{2}$, encaps.) ppm.

\section{$\mathbf{K}_{11}\left[\mathbf{C p} * \mathbf{R u}(\mathbf{1}\right.$-methylbutadiene $\left.)\left(\mathbf{H}_{2} \mathbf{O}\right) \subset \mathbf{G a}_{4} \mathbf{L}_{6}\right] \quad$ (9). ${ }^{1} \mathrm{H}$ NMR $(500 \mathrm{MHz}$,} $\left.\mathrm{D}_{2} \mathrm{O}\right)$, N-H not observed due to $\mathrm{H}-\mathrm{D}$ exchange, aromatic region broad: $1.89(\mathrm{dd}, 1 \mathrm{H},=\mathrm{C}-$ H, encaps. A), 1.63 (m, 1H, =C-H, encaps. $A$ ), 1.40 (m, 1H, =C-H, encaps. $B), 1.33$ (dd, $1 \mathrm{H},=\mathrm{C}-\mathrm{H}$, encaps. $B), 0.39\left(\mathrm{~d},{ }^{3} J=7.0 \mathrm{~Hz}, 1 \mathrm{H},=\mathrm{CH}_{2}\right.$, encaps. $\left.B\right), 0.14\left(\mathrm{~d},{ }^{3} J=7.2 \mathrm{~Hz}\right.$, $1 \mathrm{H},=\mathrm{CH}_{2}$, encaps. $\left.A\right), 0.02(\mathrm{~m}, 1 \mathrm{H},=\mathrm{C}-\mathrm{H}$, encaps. $B),-0.06\left(\mathrm{~d},{ }^{3} J=10.8 \mathrm{~Hz}, 3 \mathrm{H}, \mathrm{CH}_{3}\right.$,

encaps. $B),-0.61\left(\mathrm{~d},{ }^{3} J=6.1 \mathrm{~Hz}, 1 \mathrm{H},=\mathrm{CH}_{2}\right.$, encaps. $\left.A\right),-0.85\left(\mathrm{~d},{ }^{3} J=10.1 \mathrm{~Hz}, 3 \mathrm{H}, \mathrm{CH}_{3}\right.$, encaps. A), -0.99 (s, $15 \mathrm{H}, \mathrm{Cp}-\mathrm{CH}_{3}$, encaps $A$ ), -1.00 (s, $15 \mathrm{H}, \mathrm{Cp}-\mathrm{CH}_{3}$, encaps $B$ ), -1.96 (m, overlapping signals) ppm.

\section{$\mathbf{K}_{11}\left[\mathbf{C p} * \mathbf{R u} \mathbf{u}\right.$ (1-ethylbutadiene) $\left.\subset \mathbf{G a}_{4} \mathbf{L}_{6}\right]$ (10). ${ }^{1} \mathrm{H}$ NMR $\left(500 \mathrm{MHz}, \mathrm{D}_{2} \mathrm{O}\right), \mathrm{N}-\mathrm{H}$} not observed due to H-D exchange, aromatic region broad: $\delta 2.25\left(\mathrm{dd},{ }^{3} J=10.0 \mathrm{~Hz},{ }^{3} J=\right.$ $5.2 \mathrm{~Hz},=\mathrm{C}-\mathrm{H}$, encaps. $A$ ), $1.43\left(\mathrm{dd},{ }^{3} J=10.0 \mathrm{~Hz},{ }^{3} J=5.8 \mathrm{~Hz},=\mathrm{C}-\mathrm{H}\right.$, encaps. $B$ ), 0.77 (m, $1 \mathrm{H},=\mathrm{C}-\mathrm{H}$, encaps. $A), 0.60(\mathrm{~m}, 1 \mathrm{H},=\mathrm{C}-\mathrm{H}$, encaps. $B), 0.24\left(\mathrm{~d},{ }^{3} J=6.7 \mathrm{~Hz}, 1 \mathrm{H},=\mathrm{CH}_{2}\right.$, encaps. A), $0.20\left(\mathrm{~d},{ }^{3} J=7.7 \mathrm{~Hz}, 1 \mathrm{H},=\mathrm{CH}_{2}\right.$, encaps. $\left.B\right),-0.88$ (s, $15 \mathrm{H}, \mathrm{Cp}-\mathrm{CH}_{3}$, encaps. B), $-0.93\left(\mathrm{~s}, 15 \mathrm{H}, \mathrm{Cp}-\mathrm{CH}_{3}\right.$, encaps. $\left.A\right),-1.08\left(\mathrm{~m}, 1 \mathrm{H},-\mathrm{CH}_{2}\right.$, encaps.), $-1.15(\mathrm{~m}, 1 \mathrm{H},-$ $\mathrm{CH}_{2^{-}}$, encaps.), -1.21 (t, ${ }^{3} \mathrm{~J}=7.2 \mathrm{~Hz}, 3 \mathrm{H}, \mathrm{CH}_{3}$, encaps. $\left.B\right),-1.36\left(\mathrm{t},{ }^{3} \mathrm{~J}=7.2 \mathrm{~Hz}, 3 \mathrm{H}\right.$, $\mathrm{CH}_{3}$, encaps. $\left.A\right),-1.43\left(\mathrm{~d},{ }^{3} \mathrm{~J}=10.0 \mathrm{~Hz}, 1 \mathrm{H},=\mathrm{CH}_{2}\right.$, encaps. $\left.A\right),-1.45\left(\mathrm{~m}, 1 \mathrm{H},-\mathrm{CH}_{2}-\right.$, encaps.), $-1.71\left(\mathrm{~m}, 1 \mathrm{H},-\mathrm{CH}_{2^{-}}\right.$, encaps.), $-1.96\left(\mathrm{~d},{ }^{3} \mathrm{~J}=10.5 \mathrm{~Hz}, 1 \mathrm{H},=\mathrm{CH}_{2}\right.$, encaps. $\left.B\right)$, $2.16(\mathrm{~m}, 1 \mathrm{H},=\mathrm{C}-\mathrm{H}$, encaps. $A)$ ppm. 
$\mathbf{K}_{\mathbf{1 1}}\left[\mathbf{C p} * \mathbf{R u}(\mathbf{1}-\boldsymbol{n}\right.$-propylbutadiene $\left.) \subset \mathbf{G a}_{4} \mathbf{L}_{6}\right]$ (11). ${ }^{1} \mathrm{H} \mathrm{NMR}\left(500 \mathrm{MHz}, \mathrm{D}_{2} \mathrm{O}\right)$, N-H not observed due to H-D exchange, aromatic region broad: $\delta 2.34\left(\mathrm{dd},{ }^{3} \mathrm{~J}=10.1 \mathrm{~Hz}\right.$, ${ }^{3} J=5.8 \mathrm{~Hz},=\mathrm{C}-\mathrm{H}$, encaps. $\left.B\right), 1.86\left(\mathrm{dd},{ }^{3} J=10.6 \mathrm{~Hz},{ }^{3} \mathrm{~J}=6.8 \mathrm{~Hz},=\mathrm{C}-\mathrm{H}\right.$, encaps. $\left.A\right), 0.75$ $(\mathrm{m}, 1 \mathrm{H},=\mathrm{C}-\mathrm{H}$, encaps. $B), 0.52(\mathrm{~m}, 1 \mathrm{H},=\mathrm{C}-\mathrm{H}$, encaps. $A), 0.27\left(\mathrm{~d},{ }^{3} J=6.8 \mathrm{~Hz}, 1 \mathrm{H}\right.$, $=\mathrm{CH}_{2}$, encaps. $\left.B\right), 0.21$ (m, $1 \mathrm{H},-\mathrm{CH}_{2^{-}}$, encaps.), $0.16\left(\mathrm{~d},{ }^{3} J=7.2 \mathrm{~Hz}, 1 \mathrm{H},=\mathrm{CH}_{2}\right.$, encaps. A), $-0.07\left(\mathrm{t},{ }^{3} \mathrm{~J}=7.1 \mathrm{~Hz}, 3 \mathrm{H}, \mathrm{CH}_{3}\right.$, encaps. A), $-0.23\left(\mathrm{~m}, 1 \mathrm{H},-\mathrm{CH}_{2^{-}}\right.$, encaps.), $-0.52\left(\mathrm{t},{ }^{3} \mathrm{~J}\right.$ $=7.3 \mathrm{~Hz}, 3 \mathrm{H}, \mathrm{CH}_{3}$, encaps. $\left.B\right),-0.86\left(\mathrm{~s}, 15 \mathrm{H}, \mathrm{Cp}-\mathrm{CH}_{3}\right.$, encaps. $\left.B\right),-0.90(\mathrm{~s}, 15 \mathrm{H}, \mathrm{Cp}-$ $\mathrm{CH}_{3}$, encaps. A), -0.96 (m, $1 \mathrm{H},-\mathrm{CH}_{2^{-}}$, encaps.), -1.03 (m, $1 \mathrm{H},-\mathrm{CH}_{2^{-}}$, encaps.), $-1.32(\mathrm{~d}$, ${ }^{3} J=10.0 \mathrm{~Hz}, 1 \mathrm{H},=\mathrm{CH}_{2}$, encaps. $\left.A\right),-1.55\left(\mathrm{~m}, 1 \mathrm{H},-\mathrm{CH}_{2^{-}}\right.$, encaps.), $-1.61\left(\mathrm{~m}, 1 \mathrm{H},-\mathrm{CH}_{2^{-}}\right.$, encaps.), -1.67 (m, $1 \mathrm{H},=\mathrm{C}-\mathrm{H}$, encaps. $A),-1.84\left(\mathrm{~d},{ }^{3} \mathrm{~J}=9.9 \mathrm{~Hz}, 1 \mathrm{H},=\mathrm{CH}_{2}\right.$, encaps. $\left.B\right)$, $1.95(\mathrm{~m}, 1 \mathrm{H},=\mathrm{C}-\mathrm{H}$, encaps. $B),-2.14\left(\mathrm{~m}, 1 \mathrm{H},-\mathrm{CH}_{2^{-}}\right.$, encaps.) ppm. 


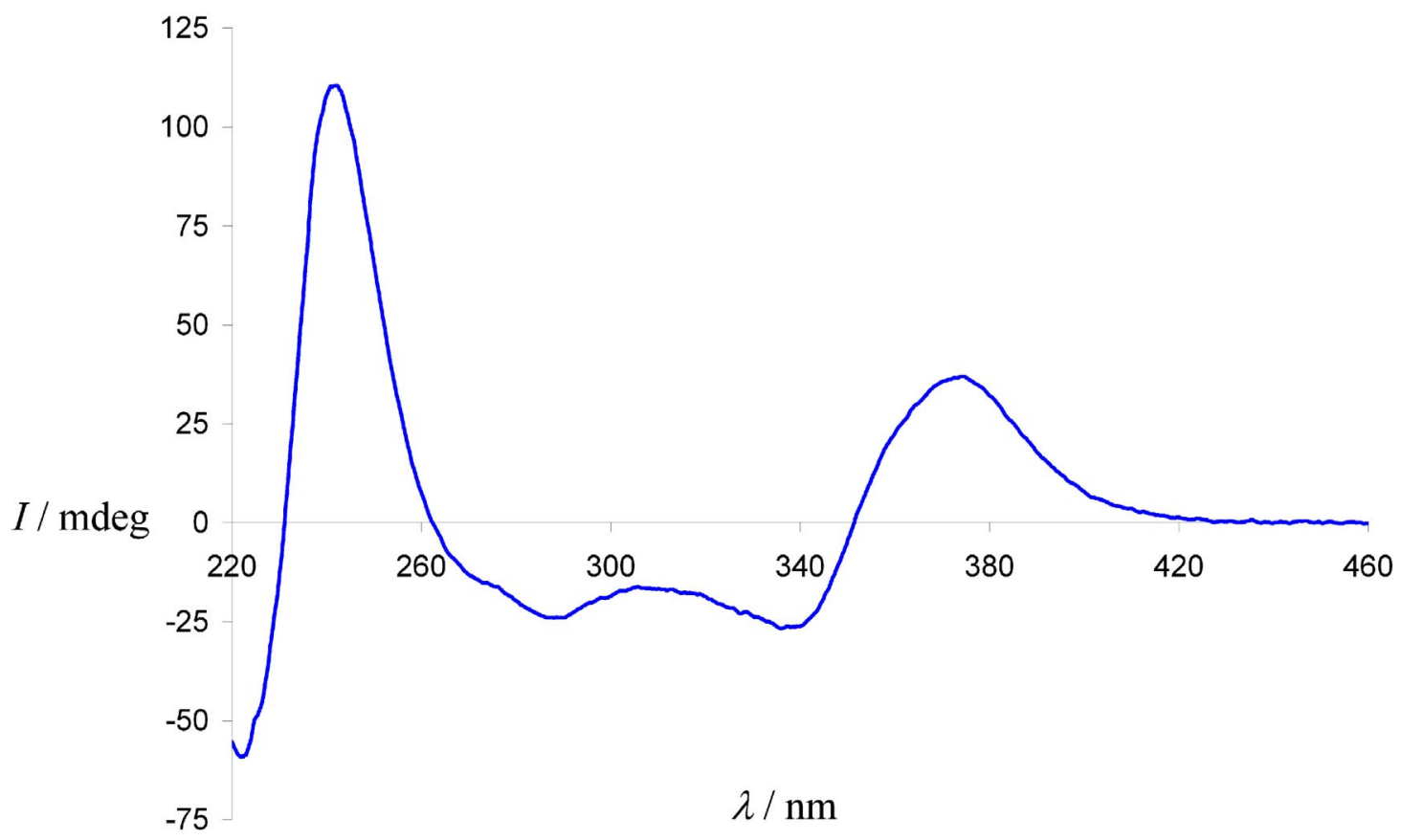

Figure 3. $\mathrm{CD}$ spectrum of the aqueous layer containing $\Lambda, \Lambda, \Lambda, \Lambda$ $[\mathrm{Cp} * \mathrm{Ru}$ (2-ethylbutadiene $\left.)\left(\mathrm{H}_{2} \mathrm{O}\right) \subset \mathrm{Ga}_{4} \mathrm{~L}_{6}\right]^{11-}(0.63 \mathrm{mM}$ in a $0.1 \mathrm{~mm}$ width cell at $20^{\circ} \mathrm{C}$ ).

\section{References}

[1] D. L. Caulder, R. E. Powers, T. N. Parac, K. N. Raymond, Angew. Chem. Int. Ed. 1998, 37, 1840-1843.

[2] M. O. Albers, D. J. Robinson, A. Shaver, E. Singleton, Organometallics 1986, 5, 2199-2205.

[3] P. J. Fagan, M. D. Ward, J. V. Caspar, J. C. Calabrese, P. J. Crusic, J. Am. Chem. Soc. 1988, 110, 2981-2983.

[4] P. J. Fagan, W. S. Mahoney, J. C. Calabrese, I. D. Williams, Organometallics 1990, 9, 1843-1852. 